\section{Images in \\ Cardiovascular Medicine}

\section{Check for updates}

\section{OPEN ACCESS}

Received: Oct 1, 2019

Accepted: Nov 19, 2019

Correspondence to

Umihiko Kaneko, MD

Department of Cardiovascular Medicine,

Sapporo Cardio Vascular Clinic, Sapporo Heart

Center, North 49, East 16, 8-1 Higashi Ward,

Sapporo 007-0849, Japan.

E-mail: uk434471@gmail.com

Copyright (c) 2020. The Korean Society of Cardiology

This is an Open Access article distributed under the terms of the Creative Commons Attribution Non-Commercial License (https:// creativecommons.org/licenses/by-nc/4.0) which permits unrestricted noncommercial use, distribution, and reproduction in any medium, provided the original work is properly cited.

ORCID iDs

Umihiko Kaneko (D)

https://orcid.org/0000-0003-2392-5084

Daisuke Hachinohe (iD)

https://orcid.org/0000-0003-4828-1836

Tsutomu Fujita (D)

https://orcid.org/0000-0002-3651-6231

Conflict of Interest

The authors have no financial conflicts of interest.

\section{Successful Use of an eSheath for Failed Introduction of the Evolut R Valve during Transfemoral Transcatheter Aortic Valve Implantation}

\author{
Umihiko Kaneko (D, MD', Ken Kobayashi, MD', Daisuke Hachinohe $\mathbb{D}, \mathrm{MD}$ ', \\ Keijiro Mitsube, MD², Azusa Furugen, MD', Takeshi Kawamura, MD³, \\ Ryuji Koshima, MD², and Tsutomu Fujita (D, MD \\ 'Department of Cardiovascular Medicine, Sapporo Cardio Vascular Clinic, Sapporo Heart Center, Sapporo, \\ Japan \\ ${ }^{2}$ Department of Cardiovascular Surgery, Sapporo Cardio Vascular Clinic, Sapporo Heart Center, Sapporo, \\ Japan \\ ${ }^{3}$ Department of Anesthesiology, Sapporo Cardio Vascular Clinic, Sapporo Heart Center, Sapporo, Japan
}

An 86-year-old man with severe, symptomatic aortic stenosis underwent a $29 \mathrm{~mm}$ Evolut $\mathrm{R}$ (Medtronic, Minneapolis, MN, USA) implantation using the right femoral artery approach. Pre-procedural computed tomography angiography showed significant tortuosity, but adequate diameters $(>7.0 \mathrm{~mm})$ and minimal calcification in both external iliac arteries (Figure 1A). After a Lunderquist Extra-Stiff Wire Guide (Cook Medical, Bloomington, IN, USA) was inserted to straighten the anatomy and increase stability, neither the 14Fr Inline nor $18 \mathrm{Fr}$ sheath could be inserted into the right iliac artery due to strong resistance. The angiography showed a severe accordion phenomenon in the right iliac artery that prevented device insertion (Figure 1B). To overcome the phenomenon, a 14Fr eSheath (Edwards Lifesciences, Irvine, CA, USA) was successfully inserted with some resistance. A $29 \mathrm{~mm}$ Evolut $\mathrm{R}$ e-Sheath was then smoothly implanted without any major vascular complications (Figure 1C-E).

An extremely tortuous iliac artery is one of the major limitations of a transfemoral approach during transcatheter aortic valve implantation, and carries the risk of serious complications including arterial dissection or rupture. To overcome the tortuosity of the access route, straightening the tortuous vessel with stiff wire is useful. ${ }^{1-3)}$ Nevertheless, after guidewire insertion, tortuous vessels (especially with little calcification) can induce an accordion phenomenon which may prevent device advancement due to the invagination of the straightened vessel wall causing severe lumen narrowing. ${ }^{4)}$ However, the InLine sheath delivery system for the Evolut R valve fit through the eSheath and was successfully implanted through a tortuous ileo-femoral vessel. 


\section{Author Contributions}

Conceptualization: Kobayashi K, Hachinohe D; Investigation: Mitsube K; Methodology: Furugen A, Kawamura T; Supervision: Koshima R, Fujita T; Writing - original draft: Kaneko U.
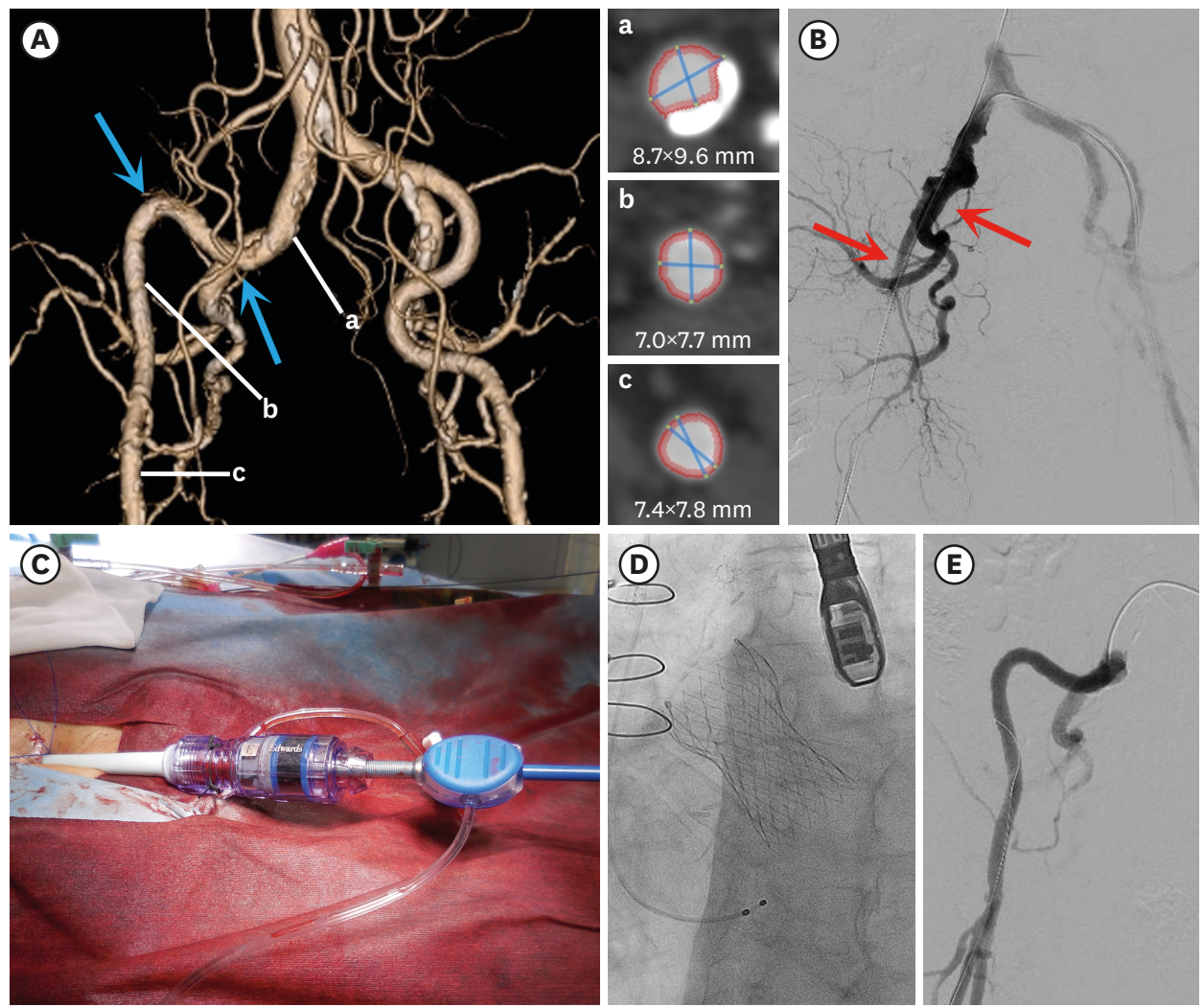

Figure 1. Computed tomographic, fluoroscopic, and intraprocedural images. (A) Pre-procedural multi-detector computed tomography revealed good caliber $(>7.0 \mathrm{~mm})$ with little calcification $(\mathrm{a}-\mathrm{c})$, but severe tortuosity in both external iliac arteries (blue arrows). (B) After stiff wire insertion, angiography showed a severe accordion phenomenon (red arrows). (C, D) Smooth delivery and implantation of a $29 \mathrm{~mm}$ Evolut $\mathrm{R}$ through the esheath was successful. (E) Final angiography confirmed no major vascular complications.

\section{REFERENCES}

1. Buzzatti N, Mangieri A, Cota L, et al. Use of double stiff wire allows successful transfemoral transcatheter aortic valve implantation through extreme thoracic aorta tortuosity. Circ Cardiovasc Interv 2015;8:e002331. PUBMED | CROSSREF

2. Kaneko U, Kobayashi K, Hachinohe D, et al. Successful direct iliac transcatheter aortic valve implantation to overcome significant tortuosity of the thoracic aorta. Korean Circ J 2018;48:949-50. PUBMED | CROSSREF

3. Naganuma T, Mitomo S, Yabushita H, Nakao T, Lazarevic A, Nakamura S. Buddy wire technique for successful transfemoral transcatheter aortic valve implantation through an extremely tortuous abdominal aorta: a basic technique in Asian patients? AsiaIntervention 2017;3:90-2.

4. Muller O, Hamilos M, Ntalianis A, Sarno G, De Bruyne B. Images in cardiovascular medicine. The accordion phenomenon: lesson from a movie. Circulation 2008;118:e677-8. PUBMED | CROSSREF 